\section{LOCOS ENTRE CUERDOS, CUERDOS EN AMBIENTES PATOLÓGICOS}

\author{
José Luis Rodríguez-Arias Palomo \\ Dr. en Psicología y Psicólogo Clínico \\ Hospital Materno-Infantil de A Coruña \\ jose.luis.rodriguez-arias.palomo@sergas.es
}

\section{THE MAD AMONG THE SANE; SANE INDIVIDUALS IN PATHOLOGIC CONTEXTS}

Cómo citar este artículo/Citation: Rodríguez-Arias Palomo, J. L. (2013). "Locos entre cuerdos, cuerdos en ambientes patológicos". Arbor, 189 (763): a069. doi: http://dx.doi.org/10.3989/ arbor.2013.763n3006

Recibido: 13 julio 2012. Aceptado: 6 junio 2013.

RESUMEN: La Salud Mental se mueve en un territorio con límites imprecisos: la consideración de un comportamiento como sano o enfermo depende del momento histórico, de la sociedad y la cultura en la que se produce o, sencillamente, de la ideología del profesional que califica ese comportamiento. Desde la perspectiva del Constructivismo Radical se exploran estos límites entre la locura y la cordura y se cuestionan los criterios de clasificación al uso en los Servicios de Salud Mental a través de una serie de ejemplos, que ponen de relieve la inestabilidad de estos y la oscuridad de los mismos. La idea que atraviesa este debate es que la autonomía de los pacientes cobra protagonismo, precisamente, en la relatividad del diagnóstico en Salud Mental, ya que, aunque "locos", tienen igual derecho que los cuerdos a decidir sobre su propia existencia y su propio estilo de vivir.

PALABRAS CLAVE: Psicodiagnóstico; Salud mental; Constructivismo; Terapia familiar.
Copyright: (C) 2013 CSIC. Este es un artículo de acceso abierto distribuido bajo los términos de la licencia Creative Commons Attribution-Non Commercial (by-nc) Spain 3.0.

ABSTRACT: Mental Health moves within a territory of uncertain boundaries: weather a behavior is considered healthy or sick depends on factors such as the historical moment, the culture and society in which the diagnosis is made or simply the professional's ideology on the matter. In this paper, the borders between madness and sanity will be explored from the point of view of Radical Constructivism (Watzlawick, 1988). The classification criteria currently used in Mental Health units will be questioned through a series of examples which reveal their instability and fuzziness. The thesis behind this debate is that the autonomy of the patient should be considered precisely in this context of relativity. Though "mad", they have equal rights to sane people to decide over their own existence and their own ways of leading their lives.

KEYWORDS: Psychodiagnosis; Mental health; Constructivism; Family therapy. 
El título de este artículo ${ }^{1}$ se inspira en el libro de Lauren Slater, "Cuerdos entre Locos: Grandes experimentos psicológicos del Siglo XX". La autora selecciona un grupo de diez experimentos psicológicos que, desde su punto de vista, han supuesto cambios, tanto en el campo de la salud mental, como en el de la vida cotidiana. Los presenta de una manera controvertida, valorando las luces y las sombras de cada uno de los experimentos que relata (Slater, 2006). Uno de los elegidos es el de David Rosenhan (1973), psicólogo americano que en los años 70 quiso comprobar el fundamento de los diagnósticos que utilizan los profesionales de Salud Mental para clasificar a los pacientes que tratan. Se le ocurrió la idea de reunir a un grupo de colegas y amigos que no tuvieran nada mejor que hacer en aquel octubre de 1972 y proponerles que se hicieran pasar por locos. Quería saber si los profesionales que tienen la responsabilidad de curar a los que enloquecen eran capaces de distinguir bien entre locos y cuerdos. Les propuso que acudieran a un dispositivo de salud mental cualquiera y simularan un único síntoma: oír una voz. Se apuntaron ocho: un pintor, un ama de casa, un estudiante de postgrado, un pediatra, un psiquiatra y tres psicólogos, uno de los cuales era el propio David Rosenhan. Para evitar interpretaciones adicionales y facilitar la homogeneidad de los síntomas entre los ocho pacientes fingidos decidieron que todos iban a decir escuchar una voz que dice "zas". Un sonido sin ningún sentido, ni significado para dar las pistas mínimas sobre el diagnóstico. No debían fingir ningún otro síntoma y debían responder a todas las preguntas que les hicieran con veracidad. Excepto en lo referente al nombre y a la profesión para no ser identificados.

Los resultados fueron contundentes: los ocho fueron ingresados. Todos, menos uno, fueron diagnosticados de esquizofrenia. El otro fue diagnosticado de psicosis maniaco-depresiva. A partir de que consiguieron su propósito de ser ingresados dejaron de manifestar el síntoma. Cuando eran interrogados respondían que ya estaban bien, que ya no oían la voz. Su estancia media en el centro hospitalario fue de 19 días -7 el que menos y 52 el que más-. Durante su vida en el hospital fueron sumisos, obedientes y buenos cumplidores. Pasaron la mayor parte del tiempo haciendo anotaciones en un cuaderno sobre la vida hospitalaria y el comportamiento de locos y profesionales. Al cabo de este tiempo fueron dados de alta por "remisión temporal de los síntomas". Ninguno fue considerado curado al final, de la misma manera que ninguno fue considerado sano al principio.
Este experimento levantó una gran polémica. Un hospital lo retó a repetirlo y enviar durante los tres meses siguientes tantos pacientes falsos como quisiera, para demostrarle que serían puntualmente reconocidos por los profesionales de salud mental de ese hospital. Al final del tercer mes el hospital informó que había detectado 41 falsos pacientes de 193 casos recibidos (21\%). Rosenhan no había enviado ninguno.

Este experimento suscita múltiples reflexiones. EI autor subraya cómo el diagnóstico en Salud Mental se hace mucho más fijándose en el contexto que en la persona. $Y$, sin embargo, el tratamiento se le ofrece a la persona porque, desde la perspectiva clásica ${ }^{2}$, se dice que son las personas las que enferman. Es a las personas a las que se les dice que se tomen tal o cual medicación, a las que se les propone tal o cual cambio de actitud o comportamiento. Y con independencia de la validez que se le quiera otorgar, lo que sí parece dejar claro es que el límite entre la cordura y la locura es borroso; que los profesionales harían bien en tomarse con prudencia los juicios clínicos que hacen, porque los diagnósticos condicionan el significado que se atribuye al comportamiento de las personas que los portan.

Las Unidades de Larga Estancia de Ingreso Hospitalario para Enfermos Mentales - así se llama ahora a los antiguos manicomios- están llenos de personas y no de contextos. Contextos solo hay uno y unifica el sentido del comportamiento de los que allí están ingresados: los locos, locos son y cualquier cosa que hagan es una locura. Y si no, ¿cómo se explica que estén ahí ingresados? Para ilustrar de qué manera los contextos condicionan el comportamiento y la interpretación que los profesionales hacen del mismo, propongo un ejemplo de la vida real y otro tomado de una ficción.

En el Cuadro 1 se incluye el documento que firman los pacientes que ingresan en una Unidad de Hospitalización (U.D.H.) para enfermos mentales que, aunque se encabece con el título de "consentimiento informado", parece más una simple nota informativa.

Resulta llamativo que una institución de ingreso voluntario esté "permanentemente cerrada". Es curioso que a los locos se les exija respeto a los horarios, a las instalaciones y a los pacientes, participación en las actividades y actitud de colaboración... ¿Cuánto tiempo tienen que portarse así para que dejen de ser considerados locos? Al mismo tiempo, se les prohíben las llamadas telefónicas y se les obliga a someterse a cuantos controles se consideren necesarios... ¿Es acaso una 


\section{CONSENTIMIENTO INFORMADO PARA EL INGRESO EN LA U.D.H.}

Para poder permanecer en esta Unidad es necesario respetar las siguientes normas de funcionamiento:

1.- El ingreso en la Unidad es voluntario y el paciente puede abandonar el tratamiento en el momento que lo solicite.

2.- La Unidad está permanentemente cerrada y el paciente no puede abandonarla sin autorización del personal sanitario.

3.- El paciente deberá respetar los horarios y participar en las actividades programadas.

4.- Tanto el tratamiento farmacológico como la duración del ingreso son competencia exclusiva del equipo terapéutico.

5.- Está totalmente prohibido consumir o portar drogas dentro de la Unidad, así como llevar objetos que puedan ser utilizados como armas.

6.- Las pertenencias del paciente podrán ser inspeccionadas en cualquier momento.

7.- Los pacientes se someterán a todos aquellos controles y pruebas analíticas que se consideren necesarias para determinar la abstinencia de drogas o por cualquier otro motivo. 8.- No está permitido ningún tipo de violencia física o verbal, manteniendo una actitud de respeto hacia el resto de pacientes y las instalaciones de la Unidad.

9.- Durante su ingreso no podrá realizar ni recibir llamadas telefónicas.

10.- Podrá recibir visitas exclusivamente de las personas autorizadas para ello por el equipo terapéutico y dentro del horario de visitas del resto del hospital.

11.- En todo momento mantendrán una actitud de colaboración y respeto con el personal sanitario.

12.- En el caso de que se incumplan las normas anteriores o que el equipo terapéutico aprecie falta de colaboración e interés, o bien se considere que la prolongación del ingreso no reporta beneficios para el paciente se procederá al alta inmediata. En el caso de que el paciente solicite el alta voluntaria o se haya procedido a la interrupción de su tratamiento por incumplimiento de las normas, no podrá volver a ingresar en la unidad hasta transcurridos al menos seis meses.

Yo,

Considero que se me han explicado y he comprendido adecuadamente las normas antes expuestas y manifiesto mi conformidad con las mismas, firmando el presente consentimiento informado.

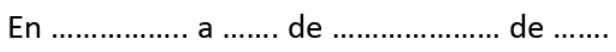

El paciente

Familiar responsable

Terapeuta 
sumisión tal compatible con la cordura? Finalmente, el incumplimiento de estas normas supone la expulsión por seis meses... ¿Significa esto que niega el tratamiento a quien sigue estando loco? ¿O más bien que la rebeldía es señal de cordura?

Y un ejemplo de ficción. En los años 80 y coincidiendo con la Reforma Psiquiátrica, uno de cuyos logros más importantes y controvertidos fue la desinstitucionalización de los pacientes que estaban ingresados en condiciones escasamente terapéuticas, Carles Mira dirigió una película - "Con el culo al aire" - en clave de humor, sobre la vida en los manicomios. El siguiente extracto corresponde a un pasaje de esa película en el que un interno veterano hace la acogida de un recién ingresado.

PAPA LUNA (Un loco disfrazado de Papa). Siéntate a mi lado y presta atención. iQué pasa! ¿No pensarás que el único cuerdo aquí eres tú?

JUAN. ¿Yo? No sé.

PAPA LUNA. Escucha. Te he estado observando y he llegado a dos conclusiones: que se puede confiar en ti y que necesitas ayuda.

JUAN. ¿Cuándo se sale de aquí?

PAPA LUNA. Se sale cuando ellos quieren, no hay reglas fijas.

JUAN. ¿Cuándo se sale de aquí?

PAPA LUNA. Ya te lo he dicho, cuando ellos quieren. De aquí se sale sin prisas. Mírame. ¿Te parezco un Papa? ¿Me ves contento?

JUAN. No sé.

PAPA LUNA. ¡Cómo que no sabes! Tú mírame bien. (Reparte bendiciones) ¿Qué? ¿No lo hago bien?

\section{JUAN. Sí.}

PAPA LUNA. ¡Claro que sí! Lo hago muy bien. Son muchos años practicando. Ya soy un hombre nuevo y cualquier día me sueltan.

JUAN. Pero yo no quiero ser Papa.

PAPA LUNA. Claro, te falta vocación. Pero tampoco querías tragarte las pastillas y te las has tragado. ¿A que sí?

\section{JUAN. Me han obligado.}

PAPA LUNA. Hummm, y a mí también. Y sin embargo, yo no me las has tragado (Hace un esfuerzo y va escupiendo las pastillas). ¿Qué te parece?

\section{JUAN. ¿Cómo lo haces?}

PAPA LUNA. Ya lo has visto, sin prisas. Aquí tienes tiempo y motivos suficientes para aprender a vomitar.

[...]

PAPA LUNA. Solo se sale de aquí cuando ellos creen que eres un hombre nuevo. $Y$ hemos descubierto que imitando a ciertos personajes históricos te llega antes la libertad.

AGUSTINA DE ARAGÓN (otro loco disfrazado de Agustina de Aragón). Cuanto mejor representes el momión escogido mejor te tratan.

PAPA LUNA. Y antes sales.

JUAN. O sea, que aquí nadie es lo que parece, ni nadie se cree nada, ni nada de nada.

PAPA LUNA. ¡Hombre, no exageres! Aquí hay de todo como en la viña del Señor.

JUAN. Pero vosotros no sois ni el Papa Luna ni Agustina de Aragón.

AGUSTINA DE ARAGÓN. Un momento, que yo sí soy Agustina de Aragón. ¿O es que no se nota?

JUAN. Pero no te lo crees.

PAPA LUNA. Escucha, Juan, no te líes. Aquí no se trata de lo que nosotros creamos o dejemos de creer, sino de lo que ellos crean. Y si ellos creen que tú crees que eres uno de sus momiones estás salvado, si no, caput.

JUAN. ¡Pero cómo se lo van a creer! ¡Es imposible!

PAPA LUNA ¿Imposible? Se nota que no los conoces. Sí se lo creen, hijo mío, sí se lo creen ¿No ves que están locos?

El pasaje es suficientemente elocuente de la locura de los contextos que los profesionales promueven como terapéuticos. ¿Es solo humor? ¿La película ya no refleja la realidad de las modernas "unidades de larga estancia para enfermos mentales" del siglo XXI?

El experimento de Rosenhan levantó ampollas y recibió múltiples críticas. Uno de sus detractores más furibundos fue Robert Spitzer (Slater, 2006), famoso psiquiatra que tiene el mérito de haber sido el Presidente del Comité de Intervención de Nomenclatura y Estadística del DSM-III (Manual Diagnóstico y Estadístico de los Trastornos Mentales). En esa tercera versión (de 1980) fue donde, definitivamente, la homosexualidad quedó desclasificada como trastorno mental. En el DSM-II la homosexualidad aparecía 
como una enfermedad mental más. En 1973 fue sustituida por "trastornos en la orientación sexual" y en 1980 se codifica como enfermedad solo la homosexualidad egodistónica, es decir, cuando el homosexual está insatisfecho con su orientación sexual y desearía cambiarla y hacerse heterosexual. En el DSM-III se argumenta así:

El punto crucial que determina si la homosexualidad per se puede ser considerada o no como un trastorno mental no reside en la etiología de esa condición, sino en sus consecuencias y en el modo en que se defina el trastorno mental. Una importante proporción de homosexuales están aparentemente satisfechos con su orientación sexual y no muestran signos ostensibles de psicopatología (a no ser que la homosexualidad en sí misma sea considerada psicopatológica), siendo capaces de actuar social y laboralmente sin ninguna dificultad. Si se emplea el criterio de malestar o incapacidad, la homosexualidad per se no es un trastorno mental. Si se recurre al criterio de desventaja inherente, no está claro en absoluto que la homosexualidad constituya una desventaja en todas las culturas o subculturas. (DSM-III, 396-397).

El comentario más inteligente que puede hacerse al respecto es el de Paul Watzlawick: "Eso ha constituido el mayor éxito jamás alcanzado, pues millones de personas se curaron de golpe de su enfermedad" (Watzlawick, 1995, 63). Robert Spitzer, no conforme con esta repentina curación de tanta gente, en la Reunión Anual de la APA (American Psychological Association) de 2001 presentó un trabajo en el que presumía de tener a su disposición un tratamiento para cambiar de forma eficaz la orientación sexual de las personas (Spitzer, 2003). De homosexual a heterosexual, por supuesto. La APA se apresuró a desaprobar el trabajo de Spitzer, arguyendo que no había suficientes pruebas para afirmar que el tratamiento propuesto por Robert Spitzer fuera eficaz para cambiar la orientación sexual.

Más recientemente y más cerca, un psiquiatra y catedrático de Psicopatología de la Universidad de San Pablo-CEU ${ }^{3}$ compareció el 20 de junio de 2005 a petición del Partido Popular ante la Comisión de Justicia del Senado para informar en relación con el Proyecto de Ley por la que se modificaba el Código Civil en materia de derecho a contraer matrimonio y, en particular, sobre los efectos que tiene en el desarrollo de los menores la convivencia con parejas homosexuales. En su ponencia, citando estudios de otros autores y basándose en su propia experiencia, afirmó que los homosexuales declaran haber tenido un padre hostil, distante, violento o alcohólico y una madre sobrepro- tectora. Precisó más: la madre es percibida por el hijo homosexual como necesitada de afecto, fría y muy exigente y por las hijas lesbianas como emocionalmente vacía. Y continuó afirmando que estos niños y niñas pueden haber sufrido en la temprana infancia abuso sexual o violación por parte de padre, madre o algún familiar. Sostuvo que en su ejercicio profesional el $30 \%$ de los homosexuales que habían pasado por su consulta habían sufrido estas experiencias. El autor no ofreció ninguna explicación del hecho de que estos hijos homosexuales y estas hijas lesbianas hubieran nacido en familias presumiblemente heterosexuales. Pero donde se pronunció más claramente fue al referirse a la comorbilidad, que él mismo describía en los siguientes términos: "Cuando dos trastornos patológicos diversos coinciden sincrónicamente en una misma persona sin que se conozcan a fondo cuáles son los grados de implicación respectiva - a veces la hay,

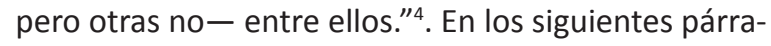
fos del discurso ante el Senado, abundó en comentarios que se referían a la homosexualidad como una patología que lleva asociada otras, como la depresión mayor, el trastorno obsesivo-compulsivo, las crisis de ansiedad generalizadas, el consumo de drogas, los trastornos de conducta y de la personalidad, con una propensión especial al trastorno de personalidad narcisista (Comisión de Justicia del Senado, 2005).

De forma análoga a lo ocurrido con Spitzer, la Universidad Complutense se apresuró a sacar un comunicado enmendando la plana a la ponencia diciendo que en la actualidad no era profesor de esa universidad y que rechazaban sus opiniones sobre la homosexualidad por considerarlas inaceptables y carentes de base científica ${ }^{5}$. De igual manera, la Sociedad Española de Psiquiatría y la de Psiquiatría Biológica emitieron también un comunicado asegurando que la comunidad científica no reconoce ningún tipo de carácter patológico en la homosexualidad.

En definitiva, de lo expuesto hasta aquí se desprende, por un lado, que para calificar a alguien como loco o cuerdo es necesario tener en cuenta la situación en la que se produce el comportamiento que se pretende calificar. Lamentablemente, el comportamiento, una vez clasificado, es considerado como propio y característico de la persona e independiente del contexto en el que se produce. Este error en la lógica del razonamiento supone una imprecisión en el juicio diagnóstico que afecta de manera trascendente a la vida de la persona que lo sufre. Si Clemente Domínguez Gómez, fundador de la Iglesia Católica Palmariana en el Palmar de Troya (Sevilla), autoproclamado Papa en 1978 
con el nombre de Gregorio XVII, no hubiera convencido a un pequeño grupo de incondicionales a los que nombró obispos y a un número nada despreciable de fieles creyentes, la sociedad le hubiera considerado un loco y como tal lo habría tratado.

Por otro lado, se sigue de lo expuesto que la consideración de alguien como loco o cuerdo depende del momento histórico y de las creencias y del criterio que utilice el profesional que lo valora y califica, al menos tanto como del comportamiento de la propia persona. Machado de Assis, en su novela "El Alienista", cuenta cómo en un pueblo de ficción - Itaguaí- el alienista -hombre de ciencia, estudioso y reflexivo- abrió una casa de locos - la Casa Verde- porque entre todas las enfermedades, consideraba que la enfermedad mental era la más desconocida. Al principio fue ingresando a quienes más o menos lo necesitaban, pero con el tiempo y fruto de sus reflexiones, llegó a la conclusión de que "la razón es el perfecto equilibrio de todas las facultades; fuera de esto insanía, solo insanía". (Machado de Assis, 1977, 47). Con este criterio ingresó a cualquiera que manifestaba el más pequeño defecto, que era tomado como signo de locura: los que se empobrecen y los que se enriquecen, los aduladores y, por supuesto, los críticos que se oponían a su criterio científico de locura, que era el verdadero. La Casa Verde se llenó de locos: "Todo era locura. Los cultivadores de enigmas, los fabricantes de charadas, de anagramas, los difamadores, los curiosos de la vida ajena, los que ponen todo su cuidado en su acicalamiento, algún que otro funcionario engreído (...) Respetaba a las enamoradas, pero no dejaba a las enamoradizas." (Machado de Assis, 1977, 94). El alienista siguió reflexionando y, en un refinamiento mayor de sus capacidades diagnósticas, llegó a la conclusión de que la perfección, la ausencia de defectos, era precisamente aquello en lo que consistía la verdadera locura: “... la verdadera doctrina no era aquella, sino la opuesta, y por lo tanto que debía admitirse como normal y ejemplar el desequilibrio de las facultades y como hipótesis patológicas todos los casos en que el equilibrio fuese ininterrumpido" (Machado de Assis, $1977,100)$. Siguiendo esta nueva directriz, puso fuera a todos los locos ingresados en la Casa Verde porque sus defectos eran la mejor prueba de cordura y empezó a ingresar a todos los ciudadanos que llevaban una trayectoria de vida ejemplar, en los que no fue capaz de descubrir defecto alguno. Y permanecían ingresados hasta que daban muestra de alguna debilidad.

Esto es una novela, pero refleja de forma preocupantemente fiel la historia de varios diagnósticos de supuestos trastornos mentales, como la homosexualidad, la esquizofrenia o el déficit de atención e hiperactividad. Los criterios que siguen quienes deciden si una persona está loca o cuerda cambian con el tiempo o en función de circunstancias e intereses, no de acuerdo a evidencias científicas. Recientemente se ha publicado en Estados Unidos - aún no en Europa- el DSM $-5^{6}$ que trae algunas novedades interesantes. En Estados Unidos ya son enfermos mentales los que comen desmesuradamente - trastorno por atracón-, o los niños que se enfadan de manera incomprensible para los adultos - trastorno de desregulación disruptiva del estado de ánimo-, o las personas que utilizan la fuerza y la coacción para obligar a otra persona a practicar sexo - trastorno parafílico- ${ }^{7}$, que han dejado de ser delincuentes para convertirse en enfermos mentales. En España y en Europa los individuos que se comportan de esa manera aún no son considerados locos, pero previsiblemente lo serán dentro de unos meses, cuando las sociedades europeas de salud mental validen el DSM-5, a no ser que se sigan utilizando los criterios $\mathrm{CIE}$, en cuyo caso habrá que esperar a que se publique la CIE-11 para saber cuál es el criterio consensuado para decidir quién está enfermo y quién no.

Todas estas consideraciones tienen importancia y afectan a la práctica clínica diaria. Hace algunos años acudió a mi consulta, derivado de otros servicios de salud mental, Vicente, un muchacho que quería ganar el Tour de Francia porque quería demostrar a todo el mundo que era capaz de hacer algo importante en su vida. Vivía con sus padres y sus dos hermanas menores en un valle de Cantabria rodeado de montañas. Desde los 17 años entrenaba todos los días, subía el Portillo de la Sía y el de la Lunada. Pedaleaba unos 150 kms diarios. Competía en campeonatos juveniles, pero sus resultados no eran todo los buenos que a él le gustaría. Su mejor puesto fue un tercero en una carrera local. Leía libros de ciclismo; se interesaba sobre todo por la preparación física de los grandes campeones, como Lance Amstrong, que fue durante años - antes de que confesara sus prácticas ilegales de dopaje- un ejemplo de superación personal: había sido capaz de ganar seis Tours de Francia seguidos justo después de haber superado un cáncer de testículo. Vicente aprendió en la literatura sobre ciclismo que se solía desaconsejar a los grandes campeones mantener relaciones sexuales dos días antes de las carreras por la pérdida de energía que, supuestamente, se produce con las eyaculaciones. Vicente dedujo que sus poluciones nocturnas podían ser las responsables de su rendimiento insuficiente. Encontró argumentos 
para amputarse un testículo, y lo hizo. Otros colegas de mi gremio calificaron su comportamiento y el razonamiento que lo soportaba como propio de un esquizofrénico. Se decidió ingresarlo en una Unidad de Agudos y se le puso tratamiento farmacológico con neurolépticos. Quince días más tarde fue dado de alta con el calificativo de "esquizofrenia desorganizada" y una pauta farmacológica para toda su vida. Tenía 19 años.

Pero Vicente, como la mayoría de los grandes campeones, era tozudo, persistente y disciplinado. Desde el primer momento se manifestó contrario a tomar la medicación - aunque la tomaba - porque le impedía rendir sobre la bicicleta y porque pensaba que los psiquiatras no habían comprendido bien las razones de su acción y por eso le habían diagnosticado erróneamente. Su psiquiatra negoció con él para que tomara la medicación durante dos años y valorarlo de nuevo al cabo de este tiempo para ver los resultados. Y así fue. Pasó los dos años diciendo que no estaba enfermo, que no quería tomar fármacos y que estos eran los responsables de no tener un rendimiento adecuado sobre la bicicleta. Al final de ese periodo Vicente decidió que ya no tomaría más medicación. Que él había cumplido su parte del trato y ahora era el psiquiatra el que tenía que cumplir la suya. Al dejar la medicación todo el mundo esperaba que se produjera un nuevo brote en cualquier momento al cabo de unas semanas. Pero no ocurrió así. Vicente estuvo trabajando duramente en el campo, ayudando a su padre en labores agrícolas y lo único reseñable de su evolución en ese período desde el punto de vista psicopatológico es que se hizo muy religioso. Tal vez excesivamente beato, pero ¿cuándo ha sido ese un motivo suficiente para catalogar a alguien como loco? A la vista de este resultado, los psiquiatras le quitaron el calificativo de esquizofrénico desorganizado y le pusieron el de pensamiento obsesivo con un nivel intelectual límite. Vicente siguió entrenando. Gastó montones de dinero en adquirir una bicicleta propia de un corredor profesional. Encargó un cuadro a su medida, luego unos pedales a su medida, más tarde un sillín a su medida y un manillar, por supuesto; pero los resultados seguían sin llegar. A veces, la frustración era tan grande que renunciaba a su objetivo y, en un arrebato, vendía su bicicleta perdiendo dinero. Unos meses más tarde volvía a comprar otra aún mejor, para ver si así conseguía su propósito. Durante esa época fue rechazado por varios equipos y varios entrenadores, de manera que tuvo que hacerse su propio programa de entrenamiento, para lo que siguió leyendo libros especializados en el tema. Cuando se hizo con una bicicleta perfectamente adaptada a sí mismo y los resultados seguían sin llegar, se dio cuenta de que él mismo tenía una serie de defectos físicos que le impedían rendir adecuadamente. Tenía el tabique nasal desviado, lo que dificultaba una respiración óptima en momentos de pleno esfuerzo; los pies planos y demasiado abiertos como consecuencia de una evidente rotación del fémur -inequívocamente confirmada por más de un traumatólogo-, por lo que la posición del pie sobre el pedal tampoco era óptima; y el pecho hundido pectus excavatum - , un examen cardiológico confirmó que por este motivo tenía el corazón ligeramente desplazado, lo que no le impedía hacer deporte y una vida normal. ¿Pero quién ha dicho que un ganador del Tour pueda hacer vida normal? La solución para los pies planos fue hacerse unas plantillas que permitieran que el pie apoyara de forma óptima sobre los pedales. Como pedía unas plantillas con un puente exagerado, en la ortopedia se negaron a modificar más sus plantillas, diciéndole que no podían hacer las plantillas que él pedía. Para la rotación del fémur la solución era quirúrgica y a base de perseverancia encontró un traumatólogo dispuesto a hacerle la operación, quien le informó de las escasas garantías del resultado y del elevado riesgo de quedar peor tras su paso por el quirófano. Pero él, con tal de ganar el Tour, estaba dispuesto a asumir cualquier riesgo. También encontró un cirujano dispuesto a operarle el pecho. El cirujano pensaba que era un problema de autoimagen y que esta mejoraría una vez que el defecto, por lo demás ostensible, fuera corregido. Y para la nariz se puso en la lista de espera de la Sanidad Pública, aunque difícilmente podía soportar la espera, ya que para ganar el Tour hay que empezar el entrenamiento adecuado en la edad adecuada, y ya tenía 21 años. En resumen, después de hacerse una bicicleta a medida, comprando cada componente en la mejor tienda del país, tenía que hacer un cuerpo a la medida de un ganador de Tour: pies, piernas, pecho y nariz.

El asunto es: ¿Todos estos razonamientos son locos o cuerdos? Porque si son locos, no puede permitirse que una persona que no está en su sano juicio tome decisiones potencial y probablemente dañinas para su salud física y también mental. Pero si son razonamientos propios de una persona cuerda, entonces ¿qué derecho tiene nadie a sustraerle la posibilidad de someterse a las intervenciones quirúrgicas que él desee y que haya, al menos, un profesional que considere que tal operación es posible y no contraria a las normas de buena práctica clínica? Cuando se me pidió que evaluara la capacidad del paciente, consulté al cirujano sobre el riesgo de la intervención para corre- 
gir el pectus excavatum. Él era favorable a realizarla, argumentando que "así, el chaval se sentiría mejor". Le pregunté si podía garantizar que Vicente ganaría el Tour de Francia.

Si se afirma que estos razonamientos son propios de un esquizofrénico entonces se le quita la capacidad de decidir en base a ellos. Si, por el contrario, se considera que Vicente no está loco, entonces hay que permitir que haga con su cuerpo lo que autónomamente quiera. ¿'Hay opciones intermedias? Por supuesto: hablar y negociar.

Pero para hablar y negociar es tan imprescindible tener clara la propia posición, como comprender y aceptar la posición del interlocutor. Es decir, negociar no es una forma de llevarse el gato al agua y demostrar que la razón está de parte de uno. Al contrario, negociar significa saber reconocer las razones del otro para que este, a su vez, sepa reconocer las nuestras y llegar a una solución de consenso, no necesariamente en un punto intermedio. El Constructivismo Radical, cuya tesis central es que la "Realidad" es una construcción en cierto modo inventada por quien la observa (von Foerster, 1991; Watzlawick, 1988) ofrece algunas soluciones.

Desde una perspectiva constructivista se distingue entre Realidad de Primer Orden: "Los sentidos nos proporcionan una imagen de la Realidad que es factible comparar con aquella percibida por otras personas, para descubrir sorpresivamente que son idénticas" (Watzlawick, 1988) y Realidad de Segundo Orden: "la que nos impide captar en forma pura sin hacer inferencias de categorizaciones, la que transforma el acto de conocimiento en subjetivo." (Ceberio y Watzlawick, 1998). La primera no es negociable: el acuerdo entre los observadores viene dado por la naturaleza y características del observador. Las personas perciben que el cielo es azul porque los ojos - los receptores - transforman el estímulo en un impulso eléctrico que cuando llega al cerebro, es interpretado de forma que todas las hablantes del español dicen: azul. No hay un solo desacuerdo. El mundo de estímulos físicos conforma la Realidad de Primer Orden. La segunda solo cobra entidad - ¿realidad? - cuando es compartida por, al menos, dos observadores. Solo "existe" lo que es validado por otra persona. Por ejemplo, un color se pone de moda cuando muchas personas quieren vestirse con ropa de ese color. El color se corresponde con una Realidad de Primer Orden, el que se ponga de moda es un acuerdo tácito de muchas personas: Realidad de Segundo Orden.
Este reconocimiento explícito de que una parte importante de lo que las personas perciben se corresponde con realidades de segundo orden es el fundamento del respeto y de la tolerancia, tanto como de la incomprensión y el desencuentro. A la vez, deja sin cabida al totalitarismo ideológico, a la dictadura de la razón y a cualquier otro tipo de totalitarismo o dictadura; porque unos y otros suponen que "Realidad" no hay más que una: la que ellos ven, defienden e imponen.

En el caso de Vicente: el pectus excavatum, el tabique nasal desviado, la rotación del fémur y los pies planos, corresponden a realidades de primer orden que pueden verificarse con las oportunas pruebas de imagen, o a simple vista, y que cualquier profesional especialista es capaz de diagnosticar sin probable desacuerdo en el diagnóstico. Ahora bien, hasta qué punto cada uno de esos defectos es o no incapacitante y la valoración que Vicente hace de los mismos, tiene que ver con su propia biografía y sus planes de vida. Se corresponde, por tanto, con una Realidad de Segundo Orden, donde caben diversas opiniones derivadas de los diferentes puntos de vista que se pueden adoptar frente a cualquier situación. ¿Hay argumentos para decir que el punto de vista de Vicente es patológico? ¿Y el de Clemente Domínguez Gómez? ¿Habría que haber ilegalizado su Iglesia Cristiana Palmariana?

De esta manera, tiene que ser Vicente quien interprete autónomamente la vida que quiere vivir y cómo quiere vivirla. Deberá ser él quien valore la importancia de los riesgos y si estos compensan o no los beneficios que se esperan. Es obligación del profesional de Salud Mental asegurarse de que Vicente cuenta con la información necesaria para fundamentar sus razonamientos; podrá opinar sobre si estos son lógicos y prudentes, si conoce y sopesa las consecuencias de sus decisiones y si está en condiciones de asumirlas.

Además, para que sus razonamientos puedan no ser considerados locos estaría bien que consiguiera que alguien más los comparta - como hizo el Papa del Palmar-, los comprenda, los asuma; en definitiva, los dé por válidos. Podría ser un entrenador que esté dispuesto a seguir con él un programa de preparación física o un club ciclista que contrate sus servicios, o un cirujano, traumatólogo, ortopedista u otorrinolaringólogo que esté dispuesto a afirmar que las peticiones de Vicente están dentro de lo que la especialidad de cada uno considera buena práctica clínica. O también un profesional de Salud Mental que informe que Vicente está en condiciones de competencia mental para decidir sobre su meta y sobre los medios que propone para conseguirla. 
La valoración de la competencia ${ }^{8}$ de una persona es un tema estudiado y controvertido (Simon, 2008; Appelbaum, Appelbaum y Grisso, 1998; Appelbaum, 2007; Drane, 1985; Roth, Meisel y Lidz, 1977) y a día de hoy no hay, ni parece probable que haya, un instrumento de medida que evalúe la capacidad de una persona de una manera objetiva; es decir, al margen o con independencia del observador que la evalúa. Es más, desde la perspectiva del Constructivismo Radical tal objetividad, ni es posible, ni es deseable. Y esto por varias razones: 1) la competencia de una persona no se puede valorar como una cualidad independiente del contexto en el que desarrolla su actividad; 2) la competencia de una persona no es una cualidad o capacidad propia, sino que solo cobra sentido cuando se valora en relación al objeto a que se destina; y 3) como se ha venido sosteniendo en este artículo, la competencia de una persona también depende de cuál sea el criterio, el punto de vista, en el que se sitúe el observador que la califica o valora. En definitiva, no se puede hablar de que una persona es o no competente en términos absolutos y permanentes, sino que su competencia solo cobra sentido cuando se dice: "es o no es competente para...".

Sí es cierto que los expertos han llegado a algunos consensos razonables para valorar la capacidad de las personas. Roth y cols. (1977) proponen cinco criterios escalonados para valorar la capacidad, que van de menos exigentes a más exigentes: 1의 La persona es capaz de hacer y expresar una elección; 2ㅇ) la persona toma una decisión razonable; 3 ㅇ) la persona toma una decisión razonable basada en argumentos racionales; 4ㅇ) La persona comprende los beneficios, riesgos y alternativas a un tratamiento; y 5ㅇ) la persona comprende todos los aspectos relevantes de la decisión a tomar y consiente de forma libre y estando bien informada. El problema es quién valora cada uno de estos cinco criterios: quién decide que una decisión es razonable, quién que está basada en argumentos racionales, quién que la persona comprende la situación... Desde esta perspectiva, cuando una persona elige una opción en contra de la opinión del profesional que la atiende, la probabilidad de que se considere una decisión poco razonable es alta y, por tanto, de que se le declare incapaz.
Y otra de las aportaciones importantes a este campo es la escala móvil de Drane (Drane, 1985). Desde esta perspectiva se reconoce de forma explícita que la capacidad es algo que está en relación directa con la decisión concreta que hay que tomar. En la valoración de la capacidad de una persona se tiene en cuenta el balance riesgo/beneficio de una opción. De esta manera se exige un grado alto de capacidad tanto para aceptar una opción que tiene más riesgo que beneficio, como para rechazar una opción que tiene más beneficio que riesgo. $Y$ al contrario, se pide un bajo nivel de capacidad tanto para aceptar una opción que tiene más beneficio que riesgo, como para rechazar algo que tiene más riesgo que beneficio.

La perspectiva del Constructivismo Radical se sitúa en el polo opuesto del "todo vale", más propio del relativismo. Si bien cualquier punto de vista de la Realidad es "no-real", es inventado, esto no significa que dé lo mismo construir una u otra Realidad o que cualquier punto de vista de la Realidad sea igualmente válido. Algunos puntos de vista se revelan eficaces para determinados propósitos y otros, en cambio, ineficaces. Algunas construcciones de realidad guían a buen puerto, otras confunden y embarrancan. Desde esta perspectiva, no es necesario oponerse a los deseos de Vicente. Bastaría con darle la oportunidad para que consiga demostrar que su punto de vista puede considerarse razonable dentro de los límites, muchas veces borrosos, característicos de estos tiempos por los que transita.

A modo de conclusión, y tomando prestadas las palabras de Paul Watzlawick, si una persona decide vivir su vida desde una perspectiva constructivista, se convertirá en una persona ante todo tolerante porque "el que llega a comprender que su mundo es su propia invención debe acordar lo mismo a los mundos de sus semejantes. (...) Además, se sentirá responsable en un sentido profundamente ético, responsable no solo de sus sueños y yerros, sino también de su mundo consciente. (...) Y plenamente libre, porque quien tiene plena conciencia de que es inventor de su propia Realidad conoce la posibilidad siempre presente de forjarla de otra manera". (Watzlawick, 1988, 265). Y podría añadirse... y absolutamente seguro de que el suelo que pisa es tan inestable como él mismo quiera construirlo. 
1 Propiamente este articulo es la versión escrita de una ponencia que tuve el privilegio de presentar en el Seminario "Fronteras Biológicas y Límites Legales: Un enfoque Bioético", organizado en noviembre de 2007 por el Departamento de Ética de la Facultad de Filosofía de la Universidad de Salmamanca. Los revisores de este artículo, a quienes agradezco el tiempo e interés que le han prestado a este texto, han visto la necesidad de actualizar las referencias y han hecho propuestas que sin duda mejoran la calidad y actualidad del texto.

2 Con la expresión "perspectiva clásica" quiero referirme al modo de pensar más extendido entre y aceptado por los profesionales de la Salud Mental actual. Profesionales que fundamentan su práctica clínica en la suposición de que es posible un saber "objetivo", al que se llega a través de los diferentes procedimientos de estudio de la "Realidad" externa al observador, que no se deforma por el propio acto observacional.

\section{BIBLIOGRAFÍA}

Appelbaum, B.C.; Appelbaum, P. S.; Grisso, T. (1998). "Competence to Consent to Voluntary Psychiatric Hospitalization: A test of a Standard Proposed by APA". Psychiatric Services, 49 (9), pp. 1193-1196.

Appelbaum, P. S. (2007). "Assessment of Patients' Competence to Consent to Treatment". N Engl J Med, 357, pp. 1834-1840.

American Psychiatric Association. (1983). DSM-III. Manual Diagnóstico y Estadístico de los Trastornos Mentales. Barcelona: Masson.

American Psychiatric Association. (2013). Diagnostic and Statistical Manual of Mental Disorders DSM-5. Arlington: Amer. Psychiatric Pub. Incorporated.

Ceberio, M. R. y Watzlawick, P. (1998). La Construcción del Universo: Conceptos introductorios y reflexiones sobre Epistemología, Constructivismo y pensamiento sistémico. Barcelona: Herder.

Comisión de Justicia del Senado (2005). Comparecencia del Catedrático de Psicopatología de la Universidad Complutense de Madrid (UCM), D. Aquilino Polaino Lorente, ante la Comisión de Justicia, para informar del Proyecto de Ley por la que se modifica el Código
3 La Presidenta de la Comisión de Justicia le presentó como Catedrático de Psicopatología de la Universidad Complutense, donde cesó su actividad en 2004.

4 http://www.senado.es/legis8/comisiones/index_S011008.html (29/8/09).

5 http://pendientedemigracion.ucm. es/cont/descargas/documento4247. pdf?pg=cont/descargas/documento4247.pdf (22/6/2005)

6 Los DSM son el consenso de la Asociación Americana de Psiquiatría (A.P.A.), en cambio la CIE es el consenso oficial de la O.M.S. Como señalan Reed y cols., "un verdadero proceso multilateral de definición de trastornos no puede ser llevado a cabo legítimamente por una única organización profesional que representa a una única disciplina dentro de la salud, en un contexto de un país concreto y

Civil en materia de derecho a contraer matrimonio $y$, en particular sobre los efectos que tiene en el desarrollo de los menores la convivencia con parejas homosexuales. Procedimiento Ordinario. Registrado el 13 de Junio de 2005, calificado el 21 de Junio de 2005. Procedimiento concluido el 20 de junio de 2005. Expediente 713000374. http:// www.senado.es/legis8/comisiones/index_S011008.html (29/8/09).

Drane, J. F. (1985). "The Many Faces of Competency". Hastings Cent. Rep, abril, pp. 17-21.

Foerster, H. von (1991). Las semillas de la Cibernética. Barcelona: Gedisa.

Machado de Assis. (1997). El Alienista. Barcelona: Tusquets Editores.

Redd, G. M.; Anaya, C. y Evans, S. C. (2012). "¿Qué es la CIE y por qué es importante en la Psicología?". International Journal of Clinical and Health Psychology, 12 (3), pp. 461-473.

Rosenhan, D. L. (1973). “On being sane in insane places". Science, 19, 179 (70), pp. 250-258.

Roth, L. H.; Meisel, A.; Lidz, C. W. (1977). "Tests of Competency to Consent to que cuenta con un interés comercial importante en los productos que desarrolla." (Reed, Anaya y Evans, 2012).

7 Para que los intereses sexuales atípicos sean considerados una patología el DSM-5 requiere como criterios que, 1) sientan angustia personal, no solo la derivada de la desaprobación social $y, 2)$ que impligue angustia para otra persona, lesión, muerte o el deseo de conducta sexuales con personas que se oponen o con personas incapaces de dar su consentimiento legal.

8 Pablo Simón discute los matices que diferencian los términos de "capacidad" y "competencia". Aquí se están utilizando como sinónimos, porque ninguna de las definiciones del ambos conceptos expresa con exactitud lo que el autor entiende por capacidad o competencia (Simón, 2008).

Trearment". Am. J. Psychiatry, 134, pp. 279-284.

Simón-Lorda, P. (2008). “La capacidad de los pacientes para tomar decisiones: una tarea todavía pendiente". Rev. Asoc. Esp. Neuropsiq., XXVIII, 102, pp. 325-348.

Slater, L. (2006). Cuerdos entre Locos. Grandes experimentos psicológicos del siglo $X X$. Barcelona: Alba Editorial.

Spitzer, R. L. (2003). "Can some gay men and lesbians change their sexual orientation? 200 participants reporting a change from homosexual to heterosexual orientation". Arch Sex Behav, 32 (5), pp. 403-17; discussion 419-72.

Universidad Complutense de Madrid (2005). Comunicado del Consejo de Dirección de la Universidad Complutense. http://pendientedemigracion.ucm. es/cont/descargas/documento 4247. pdf?pg=cont/descargas/documento4247.pdf (22/6/2013).

Watzlawick, P. (1988). La Realidad Inventada: ¿Cómo sabemos lo que creemos saber? Barcelona: Gedisa.

Watzlawick, P. (1995). El sinsentido del sentido o el sentido del sinsentido. Barcelona: Herder. 